Pleione 14(2): 301 - 309. 2020.

(C) East Himalayan Society for Spermatophyte Taxonomy

doi:10.26679/Pleione.14.2.2020.301-309

\title{
Endemic Species of Poaceae from Western Himalaya: Diversity and Distribution
}

\author{
Rekha Yadav, Shailja Tripathi, Shubham Jaiswal, Dileshwar Prasad \\ and Priyanka Agnihotri ${ }^{1}$
}

Plant diversity, Systematics \& Herbarium Division, CSIR-National Botanical Research Institute, Rana Pratap Marg, Lucknow - 226001, India

${ }^{1}$ Corresponding author: E-mail: priyagni_2006@yahoo.co.in

[Received 07.12.2020; Revised 18.12.2020; Accepted 27.12.2020; Published 31.12.2020]

\begin{abstract}
Present work deals with the distribution and diversity of endemic species of Poaceae in Western Himalaya. Out of 333 endemic taxa of Poaceae in India, 13 taxa are confined to Western Himalaya. A brief taxonomic description and type locality have been provided along with their altitudinal distribution.
\end{abstract}

Key words: Poaceae, Endemism, Western Himalaya, India.

\section{INTRODUCTION}

The term endemism is applied to those taxa that are confined to a particular geographical area and nowhere else; definitions are customized to specific needs (Heywood 1995). In recent times taxonomic units that are found exclusively in a specified geographical area i.e. peninsular regions, oceanic islands, distinct phytogeographical area or mountain peaks and isolated through ecological or temporal barriers and could be threatened due to restricted distribution are called endemic taxa. Endemic taxa are restricted to a confined region and remain separated from a widely distributed species or cosmopolitan species (Mitra \& Mukherjee 2007).

India is one of the 17 megabiodiverse countries with rich biodiversity due to its prevailing climatic, edaphic, topographic and geological stability for many years. The varied physiographic and climatic conditions have resulted in a long term stable habitat that permitted the survival of remaining tertiary flora to survive and facilitated the continuation of species diversification for the evolution of new species, thereby leading to a greater number of endemic plants. The degree of endemism for an area is often cited as a measure of the uniqueness of the flora and consequently is important for prioritizing sites for conservation (Myers et al. 2000).

The members of Poaceae Barnhart are commonly known as grasses. It is nested in the order Poales along with 13 other families and is the $5^{\text {th }}$ largest plant family in the world. However, it stands first in its economic and ecological importance (Hodkinson 2018). Although family shows cosmopolitan distribution inhabiting in all continents, including Antarctica (Convey 2001) but some species are confined to a particular area. In India, Poaceae comprises of about 1300 species belonging to 268 genera (Karthikeyan et al. 1989). Amongst the total angiospermic flora of India, Poaceae has the largest number of endemic taxa, contributing around $7.75 \%$ of the angiosperm taxa (Singh et al. 2018). Chatterjee (1939) pioneered the studies on endemic plants of India. He reported 6580 endemic dicots to this region, representing $61.5 \%$ of Indian flora. Of these, 3169 species were endemic to Himalayan region and 2045 species were endemic to Peninsular India. Many naturalists and botanists have also surveyed the endemic plant taxa in India (Nayar 1996; Karthikeyan 2000; Ahmedullah 2000; Mitra \& Mukherjee 2007; Irwin \& Narasimhan 2011). Dhar et al. (2012) reported 153 endemic taxa to Kashmir Himalaya including 


\section{Endemic species of Poaceae from Western Himalaya}

six endemic taxa of Poaceae. Recently, Singh $e t$ al. (2015) enumerated only 86 genera including 335 taxa as strict endemics to India, of which, 15 taxa are reported from Western Himalaya. During our revisionary studies on Poaceae from Western Himalaya, we came across with three additional endemic taxa viz. Agropyron thomsonii Hook.f., Festuca lucida Stapf, and Poa garbwalensis D.C. Nautiyal \& R.D. Gaur that were not earlier reported earlier by Singh et al. (2015). Phytogeographically, Western Himalaya refers to the westernmost section of the Great Himalayas, which stretch across northeastern Afghanistan through India to central Nepal (Tripathi et al. 2019). In India, Western Himalaya stretches between 28p 452 - 36p $202 \mathrm{~N}$ and 73p 262 - 80p 242 E covering an area of ca. 331,402 $\mathrm{km}^{2}$ and passes through three states viz., Jammu \& Kashmir, Himachal Pradesh and Uttarakhand (Jalal \& Jayanthi 2015) (Figure 1). The Western Himalaya comprises of about $10 \%$ of the country's total geographical area (Jalal \& Jayanthi 2015). Due to habitat diversity along with rich biodiversity, Western Himalaya has always been a fascinating site for the taxonomists for observation of biodiversity. Based on extensive literature survey, nomenclatural changes and extended distributional range, the present paper attempts an updated analysis of taxa of Poaceae that are endemic exclusively to the Western Himalayan region.

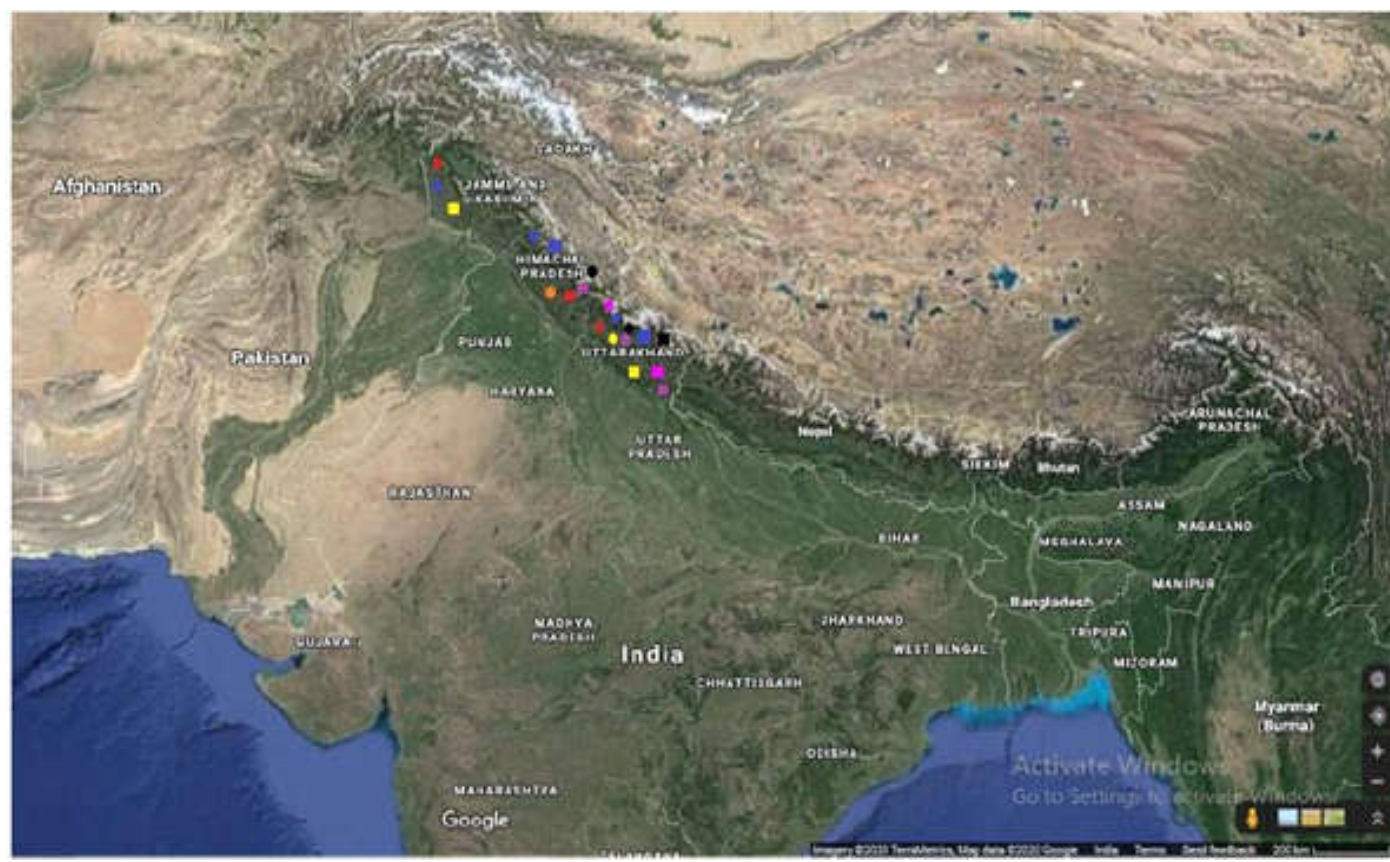

- Agropyron thomsonit

Dendrocalamus somdevae

- Eulalia madkotiensis

- Festuca lucida

- Festuca nandadevica

- Festuca sanjappae

- Helictotrichon uniyalii a Muhlenbergia rakhchamensis

Poa garhwalensis

a Poa rhadina

- Pseudodanthonia himalaica

- Sehima notatum

- Koeleria micans

Figure 1. Map of Western Himalaya having endemic species in its three states viz., Jammu and Kashmir, Himachal Pradesh, and Uttarakhand. 
TAXONOMIC TREATMENTS

Agropyron Gaertn.

Agropyron thomsonii Hook.f., Fl. Brit. India (J.D. Hooker). 7: 370. 1896.

Type: Western Himalaya, alt. 3000 - 3700 m from Kunawur and Piti; Jacquemont s.n., Thomson s.n. Garhwal; R. Strachey \& J.E. Winterbottom s.n., Dutbie s.n.

Perennial herbs. Culms $60-90 \mathrm{~cm}$ tall, slender. Leaf-blade flat, upper surface glabrous, lower more or less hairy. Racemes $7.6-15.2 \mathrm{~cm}$ long. Spikelets $0.6-1.5 \mathrm{~cm}$ long, awnless, 5 - 7flowered, scaberulous, ovoid, erect; lower glumes and upper glume ovate to oblong, hyaline, 5-7- nerved, apex acute or acuminate sometimes upper glume apex toothed and mucronate; lemma ovate to lanceolate, 5-nerved, villous; palea keels ciliate. Stamens 3.

\section{Dendrocalamus Nees}

Dendrocalamus somdevae H.B. Naithani, Indian For. 119(6): 504 - 506. 1993.

Type: Uttarakhand, Dehra Dun, Haridwar Road between Jogiwala and Majri (Mokhampur), 11 March 1991, Somadeva 10985 (Holotype: DD).

Perennial herbs, pachymorphic, short rhizomes. Culms woody upto $20 \mathrm{~m}$ tall. Culm sheath $35-45 \mathrm{~cm} \times 18-20 \mathrm{~cm}$, imperfect blade ovate to lanceolate upto $30 \mathrm{~cm}$ long; ligule entire or dentate, sometimes fimbriate. Leaf-blade lanceolate, $37.5 \mathrm{~cm} \times 3.75 \mathrm{~cm}$, acuminate, scabrous and twisted, margins finely serrate, base rounded with short thick petiole; leaf-sheath keeled, outer surface hairy, inner surface glabrous; ligule obliquely truncate. Panicles bearing half-verticillate semi-globular heads of purple spikelets. Spikelets $0.8-1.5 \mathrm{~cm} \times 0.3-0.5 \mathrm{~cm}, 2-3$-flowered; lemma outer surface glabrous, inner surface hairy, margins ciliate; palea two-keeled, ciliate. Stamens 6; caryopsis stony with short beack at apex.

\section{Eulalia Kunth}

Eulalia madkotiensis Kandwal, B.K. Gupta \& S.K. Srivast., Kew Bull. 62: 519. 2007.

Type: India, Uttarakhand, Pithoragarh Distt, Madkot, towards Jauljibi, 2 Aug. 2004, Manish K. Kandwal, 3363 (Holotype: BSD).

Perennial herbs. Culms terete up to $50 \mathrm{~cm}$ high. Leaf-blade $25-50 \times 0.2 \mathrm{~cm}$, hairy, filiform; leaf-sheath pubescent; ligule membranous. Racemes digitate upto $9 \mathrm{~cm}$ long. Spikelets elliptic, paired, similar, sessile and pedicelled; lower glume 2-nerved, $1.6 \mathrm{~mm}$ long pilose hairs, awns 2, subequal; upper glume compressed, awned. Lower floret empty; lemma oblanceolate, c. $3 \mathrm{~mm}$ long, acute; palea absent. Upper floret hermaphrodite; lemma c. $3 \mathrm{~mm}$ long, hyaline, awned; palea ovate upto $2 \mathrm{~mm}$ long. Lodicules 2, cuneate, truncate. Stamens 3, c. 2 mm long.

\section{Festuca L.}

Festuca lucida Stapf, Fl. Brit. Ind. 7: 355. 1896.

Type: Uttarakhand, Jaunsar, Deodar forest below Karambar Peak, 2700 m, 03 May 1894, Duthie 14481 (Lectotype: K, K000032138!, designated by Kar 2017: 69).

Perennial herbs. Culms erect, $0.6-1 \mathrm{~m}$ tall, glabrous. Leaf-blade $15-30 \mathrm{~cm} \times 1.2-1.8 \mathrm{~cm}$, flat, scaberulous, glabrous, acuminate; ligule membranous, $5-9 \mathrm{~mm}$ long, lacerate; leaf-sheath glabrous, $20-24 \mathrm{~cm}$ long. Panicles lax, $15-20 \mathrm{~cm} \times 0.7-0.9 \mathrm{~cm}$. Spikelets single, laterally compressed, $8-15 \mathrm{~mm} \times 2.1-2.6 \mathrm{~mm}$, acute; lower glume 1-nerved, $3-5 \mathrm{~mm} \times 1.3-1.8$ $\mathrm{mm}$, ovate to lanceolate, glabrous, acute; upper glume 3-nerved, $4-6 \mathrm{~mm} \times 2-2.1 \mathrm{~mm}$, ovate to lanceolate, glabrous, apex acute; lemma 5-nerved, $6-9.1 \mathrm{~mm} \times 2-3 \mathrm{~mm}$, oblong 
to lanceolate, awnless; palea 2-keeled, oblong to lanceolate, $6-7 \mathrm{~mm} \times 1-1.2 \mathrm{~mm}$, hyaline, apex acute and bi-fid. Lodicules 2, membranous, $1-1.1 \mathrm{~mm} \times 0.2-0.4 \mathrm{~mm}$. Stamens 3, 7 $-4 \mathrm{~mm} \times 0.2-0.4 \mathrm{~mm}$.

Festuca nandadevica Hajra, Indian J. Forest. 6(1): 79 - 80. 1983.

Type: Uttarakhand, Chamoli distt, Deodi-Ramani, Nanda Devi National Park, Hab. In Rhododendron-Betula Forest 25.08.1981, Hajra 73285A (Holotype: CAL, CAL0000002492!).

Perennial herbs. Culms erect, glabrous upto $30-70 \mathrm{~cm}$ tall. Leaf-blade linear to lanceolate, 3 - $18 \mathrm{~mm} \times 1.5-3 \mathrm{~mm}$, pubescent; ligule membranous, eciliate, $1-2 \mathrm{~mm}$ long, auricles minute, erect; leaf-sheath $10-14 \mathrm{~cm}$, glabrous. Panicles nodding, $25-30 \mathrm{~cm} \times 1-3 \mathrm{~cm}$. Spikelets solitary, pedicelate, laterally compressed, $8-12 \times 1.5-1.8 \mathrm{~mm}$; glumes dissimilar; lower glume 1-nerved, lanceolate, $3-4 \times 0.8-1.6 \mathrm{~mm}$, acuminate; upper glume 1-nerved, lanceolate, $3-4 \times 0.5-0.8 \mathrm{~mm}$; lemma 5 -nerved, $7-8 \times 0.8-1 \mathrm{~mm}$, ovate to lanceolate, awned; palea 2-keeled, $7-8 \times 0.8-1 \mathrm{~mm}$, apex bifid. Lodicules 2, membranous, $1.1-1.3$ $\times 0.2-0.4 \mathrm{~mm}$. Stamens 3, $1.5-2 \times 0.2-0.5 \mathrm{~mm}$.

Festuca sanjappae Chandra Sek. \& S.K. Srivast., J. Jap. Bot. 80: 72. 2005.

Type: Himachal Pradesh, Pin Valley National Park, Chhohem, 4000 - 4100 m.; K. Chandra Sekar 103271 (Holotype: CAL).

Perennial herbs. Culms terete, $35-43 \times 1-2.5 \mathrm{~mm}$, hairy. Leaf-blade, flat, 7-nerved, sparsely hairy, $6-10.2 \times 3-4 \mathrm{~mm}$; ligule membranous, $1-1.4 \mathrm{~mm}$ long. Panicles elliptic to oblanceolate $6.5-14 \times 5-2.5 \mathrm{~cm}$. Spikelets $4-5$ flowered, ovate-lanceolate or elliptic ovate, $4-5 \times 1.8$ - $2.2 \mathrm{~mm}$; lower glume 1-nerved, $3.7-4 \times 0.8-0.9 \mathrm{~mm}$; upper glume 3-nerved, $4-4.5 \times$ $0.9-1 \mathrm{~mm}$; lemma ovate or elliptic-lanceolate, slightly bifid at tip, $3.9-4.2 \times 0.9-1.1 \mathrm{~mm}$, awned; awn $1.4-1.6 \mathrm{~cm}$ long; palea 2-keeled, lanceolate, $3.2-3.8 \times 0.5-0.8 \mathrm{~mm}$. Lodicules 2, elliptic to obovate $0.2-0.35 \times 0.1-0.2 \mathrm{~mm}$. Stamens 3, $0.2-0.6 \mathrm{~mm}$ long.

\section{Helictotrichon Besser}

Helictotrichon uniyalii Kandwal \& B.K. Gupta, Nordic J. Bot. 28: 47 - 48. 2010.

Type: Uttarakhand, Distt Bageshwar, near Dhakuri, 20 Sep 2003, Manish K. Kandwal 1186 (Holotype: BSD).

Perennial herbs. Culms, erect, upto $40-60 \mathrm{~cm}$ tall. Leaf-blade $15-23 \times 0.2 \mathrm{~cm}$, conduplicate; ligule membranous upto $1.2 \mathrm{~mm}$ long. Panicles $7.5-16 \times 1-2 \mathrm{~cm}$, erect, branches ascending. Spikelets 2 - 3-flowered, laterally compressed, $5.5-7.5 \mathrm{~mm}$ long; lower 2 fertile flowers, bisexual, upper floret rudimentary; lower glume 1-nerved, linear-lanceolate, upto $6.6 \mathrm{~mm}$ long; upper glume 3-nerved lanceolate, 6.0 - $7.0 \mathrm{~mm}$ long; lemma 5-nerved lanceolate, 5.5 $7.0 \mathrm{~mm}$ long, apex bifid, awned; awn scabrid with twisted column; palea linear, 2-keeled, keels scabrous, 5.0 - $5.5 \mathrm{~mm}$ long. Lodicules 2. Stamens 3, upto $2 \mathrm{~mm}$ long.

\section{Koeleria Pers.}

Koeleria micans (Hook.f.) Barberá, Quintanar, Soreng, \& P.M. Peterson, Phytoneuron 46:1 13. 2019. Avena micans Hook.f., Fl. Brit. India 7: 279. 1896.

Type: Uttarakhand, Garhwal division, Tehri Garhwal, 30³0’ N 78³0' E, J.F. Duthie 46 (Lectotype: K, K000032268!, designated by Barberá et al. 2019: 5).

Perennial herbs. Culms $40-50 \mathrm{~cm}$ heigh, tufted. Leaf sheath glabrous; ligule membranous, lacerate; leaf blade $15-30 \mathrm{~cm}$ long, flat, glabrous or scaberulous, apex finely acuminate. Panicles $7-10 \mathrm{~cm}$ long, erect or inclined. Spikelets laterally compressed, $6-7 \mathrm{~mm}$ long; lower glume lanceolate, 1-nerved; upper glume 1-nerved, lanceolate to oblong; lemma 5nerved, apex acuminate and bifid, awned; awn straight or recurved; palea 2-keeled, keels minutely scabrid. Lodicule 2 . Stamens 3 , linear. 


\section{Muhlenbergia Schreb.}

Muhlenbergia rakhchamensisArum., G.V.S. Murthy \& V.J. Nair, Indian J. Forest. 36(1): 51 54. 2013.

Type: Himachal Pradesh, Kinnaurdistt, Baspa Valley, Rakhcham, 2950 m, 13.8.1973, K.P. Janardhanan, 52661 (Holotype: CAL).

Tufted annual herbs. Culms erect, $25-35 \mathrm{~cm}$ tall. Leaf-blade flat, linear to lanceolate, $0.5-1.7$ $\times 0.2-0.4 \mathrm{~cm}$; leaf sheath $0.6-4.1 \mathrm{~cm}$ long, glabrous; ligule membranous, $0.3-0.5 \mathrm{~mm}$ long, apex lacerate or fimbriate. Panicles $1.5-4 \times 0.3-0.5 \mathrm{~cm}$, scabrous. Spikelets solitary, 2 $3 \times 0.5-1 \mathrm{~mm}$, flowers 2 . Lower floret hermaphrodite. Upper floret hermaphrodite sometimes male or sterile. Lower glume 1-nerved, glabrous, lanceolate, $1.9-2.3 \times 0.5 \mathrm{~mm}$, acute or aristate; upper glume 1-nerved, elliptic to lanceolate, $2-2.3 \times 0.6 \mathrm{~mm}$, apex muticous or acute. Florets oblong to lanceolate, $2-2.8 \times 0.5-0.8 \mathrm{~mm}$; lemma 3 or 5 -nerved, lanceolate, $2-2.5 \times 0.3-0.8 \mathrm{~mm}$, awned; awn scaberulous, $2-7 \mathrm{~mm}$ long; palea 2-keeled, elliptic to lanceolate, $2-2.5 \times 0.3-0.6 \mathrm{~mm}$, acute or acuminate. Lodicules 2 upto $0.3 \mathrm{~mm}$ long. Stamens 3 upto $1 \mathrm{~mm}$ long.

\section{Poa L.}

Poa garhwalensis D.C.Nautiyal \& R.D.Gaur, J. Bombay Nat. Hist. Soc. 96: 285 - 287. 1999. Type: Uttrarakhand, Chamoli distt., Leptal, 4000 m, 4 Aug. 1996, D.C. Nautiyal 13501A (Holotype: GUH).

Perennial herbs. Culms erect, $45-60 \mathrm{~cm}$ tall, glabrous. Leaf-blade $4-10 \times 0.2-0.3 \mathrm{~cm}$, linear, acute; leaf-sheath keeled, $10-20 \mathrm{~cm}$ long; ligule $1.6-3.5 \mathrm{~mm}$ long, ovate. Panicles lax, $3.5-$ $10 \times 3-4 \mathrm{~cm}$, scabrid. Spikelets $4-12 \mathrm{~mm}$ long, elliptic; lower glume, nerves 3, 3-3.2 mm long, oblong, smooth, apex acute; upper glume 3 -nerved, $3.0-4.0 \mathrm{~mm}$ long, oblong, glabrous; lemma 5-nerved, $4-4.8 \mathrm{~mm}$ long, scabrid; palea $2.8-4.5 \mathrm{~mm}$ long, oblong to elliptic. Stamens 3, $2-2.5 \mathrm{~mm}$ long.

Poa rhadina Bor, Kew Bull. 3(1): 138 - 144. 1948.

Type: Uttarakhand, Tehri-Garwahal, Jaulea bah, Srikanta, 3700 - 4000 m., 7 Aug 1883, J. F. Duthie 265 (Holotype: K).

Annual herbs. Culms erect, $6-16 \mathrm{~cm}$ tall. Leaf blade linear, scabrous, $3-5.5 \times 0.1 \mathrm{~cm}$, acuminate. Leaf sheath glabrous; ligule eciliate, $2-3 \mathrm{~mm}$ long. Panicles elliptic to oblong, 2.5 $-6.5 \times 1.5-3 \mathrm{~cm}$, branches scabrid and spreading. Spikelets wedge-shaped, $2.5-3 \mathrm{~mm}$ long, florets $2-3(-4)$; lower glume oblong, 3-nerved, $2.5-2.7 \mathrm{~mm}$ long, acuminate; upper glume oblong, 3-nerved, 2.7 - $3 \mathrm{~mm}$ long, acute; lemmas 5-nerved, 2 - $2.5 \mathrm{~mm}$ long, apex obtuse; palea oblong to elliptic, $1.7 \mathrm{~mm}$ long, keels scabrid. Stamens $0.5-0.6 \mathrm{~mm}$ long.

\section{Pseudodanthonia Bor \& C.E. Hubb.}

Pseudodanthonia himalaica (Hook.f.) Bor \& C.E. Hubb., Kew Bull. 12(3): 425 - 427. 1957 publ. 1958. Danthonia himalaica Hook.f., Fl. Brit. India 7: 281. 1896.

Type: Uttarakhand,Tehri Garhwal, Jaunsar, rocks on east side of Lakandi Peak, 2500 - 2800 m, 19 Apr. 1894, J. F. Duthie 14467 (Holotype: K).

Perennial herbs. Culms upto $45 \mathrm{~cm}$ tall. Leaf blade involute, 15 “ $30 \mathrm{~cm} \times 1.5$ “ $2.0 \mathrm{~mm}$. Panicles contracted, lanceolate, $2.0 " 9.0 \mathrm{~cm} \times 0.5$ " $1.0 \mathrm{~mm}$. Spikelets solitary; cuneate, laterally compressed, 1.2 “ $2.5 \mathrm{~cm}$ long. Fertile spikelets pedicelate, pedicels ciliate; glumes similar; lemma 7 - 9-nerved, lanceolate, 10 - 14 mm long, apex bifid, awned; palea winged, 2-keeled. Lodicules 2. Stamens 3. 


\section{Sehima Forssk.}

Sehima notata (Hack.) A. Camus., Bull. Mus. Hist. Nat. 27:372 - 373. 1921. Ischaemum notatum Hackel, Mono. Phanero. 6: 246 - 247. 1889.

Type: Uttarakhand, Almoradistt, Dunagiri mountain, 1800 - 2100 m, 28 September 1885, J.F. Duthie 5057 (Lectotype: K, K000245768!, designated by Tiwari et al. 2018: 95).

Perennial herbs. Culms $50-120 \mathrm{~cm}$ tall. Leaf-sheaths glabrous or hispid. Ligule ciliate; leaf-blades flat, hairy, $10-40 \times 0.3-0.6 \mathrm{~cm}$, acuminate. Racemes $9-15 \mathrm{~cm}$ long. Spikelets appressed, paired, sessile and pedicelate; lower glume 8-nerved, lanceolate, apex awned, bifid, dentate; upper glume lanceolate, 7-nerved, apex acute, awned, awn $10-11$ $\mathrm{mm}$ long; lemma lanceolate, 3-nerved, 40 - $45 \mathrm{~mm}$ long, apex bifid, awned; palea hyaline. Stamens 3, 4-5 mm long.

\section{RESULT AND DISCUSSION}

The present study revealed that a total of 13 species nested within 10 genera of Poaceae are endemic to Western Himalayan region including Agropyron thomsonii, Dendrocalamus somdevae, Eulalia madkotiensis, Festuca lucida, F. nandadevica, F. sanjappae, Helictotrichon uniyalii, Koeleria micans, Mublenbergia rakhchamensis, Poa garbwalensis, P. rhadina, Pseudodanthonia bimalaica and Sebima notatum (Table 1). Occurrence of taxa ranges between the elevations of ca. $1000-5180$ m. Sehima notatum is known to possess a wide distributional range along the altitudinal gradient. Distribution

Table 1. Distribution of endemic taxa of Poaceae in Western Himalaya with its altitudinal range [Abbreviations used: $\mathrm{J} \& \mathrm{~K}=\mathrm{Jammu}$ and Kashmir; HP $=$ Himachal Pradesh; UK = Uttarakhand].

\begin{tabular}{|l|l|l|l|}
\hline $\begin{array}{l}\text { Sr. } \\
\text { No. }\end{array}$ & Name of Taxa & $\begin{array}{l}\text { Altitude } \\
(\mathbf{m})\end{array}$ & States \\
\hline 1. & Agropyron thomsonii & $3048-3657$ & J\&K, UK \\
\hline 2. & Dendrocalamus somdevae & 1000 & UK \\
\hline 3. & Eulaliamadkotiensis & 1500 & UK \\
\hline 4. & Festuca lucida & $2133-5180$ & J\&K, HP, UK \\
\hline 5. & Festuca nandadevica & $3500-3550$ & HP, UK \\
\hline 6. & Festuca sanjappae & $4000-4100$ & HP \\
\hline 7. & Helictotrichonuniyalii & 1004 & UK \\
\hline 8. & Koeleria micans & $3000-4100$ & HP \& UK \\
\hline 9. & Mublenbergia rakhchamensis & 2950 & HP \\
\hline 10. & Poa garbwalensis & 4000 & J\&K, UK \\
\hline 11. & Poa rbadina & $3500-5000$ & UK \\
\hline 12. & Psendodanthonia bimalaica & $2100-2150$ & HP, UK \\
\hline 13. & Sebima notatum & $1600-4000$ & UK \\
\hline
\end{tabular}

of the taxa reveals that out of 13 taxa endemic to Western Himalaya, 41\% taxa are found exclusively in Uttarakhand and 16\% in Himachal Pradesh (Figure 2). Assessment of the state wise distribution of endemic taxa revealed that about $80 \%$ of total endemic grasses from Western Himalaya have been recorded from tropical to alpine region of Uttarakhand. Therefore, 


\section{Degree of Endemism in different states of Western Himalaya}

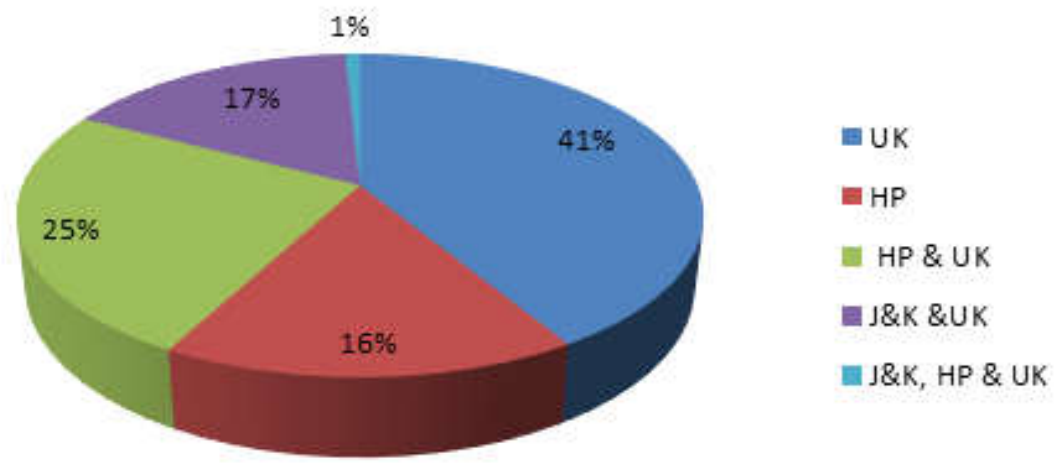

Figure 2. Degree of Endemism in different states of Western Himalaya.

Uttarakhand provides optimum conditions for the vegetation of grasses. Restriction of these 13 endemic taxa to Western Himalaya may be attributed due to their habitat specificity. Due to restricted geographical distribution, habitat specificity, endemic taxa are more vulnerable to natural and anthropogenic threats and, therefore, hold a high risk of extinction. Endemic plants are of substantial phytogeographic value. Being the basic component of any flora, they determine the degree of uniqueness of that region. Therefore, endemic species should be closely monitored and managed, and assessment of their conservation also be considered a global priority.

\section{Acknowledgements}

Authors are thankful to the Director, CSIR-NBRI, Lucknow for encouragement and facilities. Thanks are also due to Dr. Tariq Husain, ex- Senior Principal Scientist, CSIR- NBRI, Lucknow for his consistent guidance. First, Second and Fourth authors acknowledge the CSIR, New Delhi, and third author to the UGC, New Delhi for granting research fellowship to carrying out the research work.

\section{LITERATURE CITED}

Ahmedullah, M. 2000. Endemism in the Indian flora. In: Flora of India (Introductory volume), Part-II. (eds. N.P. Singh et al.,) Botanical Survey of India, Calcutta. pp $246-265$.

Arumugam, S.; Murthy G.V.S. \& Nair, V.J. 2013. Mublenbergia rakhchamensis (Poaceae): A new species from Himachal Pradesh, India. Indian J. Forest. 36(1): 51 - 54.

Bor, N.L. 1948. New species of Poa from India, Burma and Tibet. Kew Bull. 3(1): 138 - 144.

Bor, N.L. \& Hubbard, C.E. 1957. Notes on Asiatic Grasses: XXXI. A New Genus of Grasses. Kew Bull. 12(3): 425 - 427.

Camus, A. 1921. Note sur les esUecesasiatiques du genre SehimaForsk. Bull. Mus. Hist. Nat. 27: $372-373$.

Chatterjee, D. 1939. Studies on the endemic flora of India and Burma. J. Asiat. Soc. Bengal. Sci. 5(1): $1-573$.

Convey, P. 2001. Antarctic ecosystems. In: Levin, S.A. (ed.) Encyclopedia of biodiversity. San Diego: Academic press. Pp. $171-184$. 
Dar, G.H.; Khuroo, A.A. \& Nasreen, A. 2012. Endemism in the angiosperm flora of Kashmir Valley, India: Stocktaking. In: S.K. Mukherjee \& G.G. Maiti (eds.), Proceedings of International Seminar on "Multidisciplinary Approaches in Angiospenn Systematics", University of Kalyani, Kalyani.

Hackel, E. 1889. Andropogoneae. In: De Candolle, A. \& De Candolle, C. (Eds.) Monographiae phanerogamarum, vol. 6. Sumptibus G. Masson, Paris, pp. 716

Hajra, P.K. 1983. A new species of Festuca (Poaceae) from Nandadevi National Park, Chamoli District, Uttarpradesh. Indian J. Forest. 6(1): $79-80$.

Heywood, V. H. 1995. Global Biodiversity Assessment. Published for the UNEP, Cambridge University Press.

Hodkinson, T.R. 2018. Evolution and Taxonomy of the Grasses (Poaceae): A Model Family for the study of Species- rich groups. Ann. Plant Rev.1. Pp. 1-39.

Hooker, J.D. 1896. The Flora of British India 7. L. Reeve and Co., London, pp. 1-842.

Hooker, J.D. 1904. A Sketch of the Flora of British India. Eyre and Spottiswoode, London.

Irwin, S. J. \& Narasimhan, D. 2011. Endemic genera of Angiosperms in India: A Review. Rheedea 21(1): 87-105.

Jalal J.S. \& Jayanthi, J. 2015. An annotated checklist of the orchids of Western Himalaya, India. Lankesteriana 15(1): 7 - 50.

Kandwal, M.K.; Gupta, B.K. \& Srivastava, S.K. 2007. A New Species of Eulalia (Poaceae) from India. Kew Bull. 62 (3): 519 - 521.

Kandwal, M.K. \& Gupta, B.K. 2010. Helictotrichonuniyaliisp.nov. from Uttarakhand (India). Nord. J. Bot. 28: $47-48$.

Kar, S. 2017. Taxonomic Revision of the genus Festuca L. (Poaceae) in India. Ph.D. thesis, University of Calcutta, Kolkata. Retrieved from http://hdl.handle.net/10603/248261.

Karthikeyan, S.; Jain, S.K.; Nayer, M. P. \&Sanjappa, M. 1989. Florae Indicae Enumeration: Monocotyledonae. Botanical Survey of India. Calcutta.

Kumar, M.S.M. 2009. Revisionary Studies on four genera of Indian Bamboos. Kerala Forest Research Report, Kerala, India.

Mitra, S.; Mukherjee, S.K. 2007. Reassessment and diversity of endemic angiospermic genera of India. J. Econ. Taxon. Bot. 31(1): 163 - 176.

Myers, N.; Mittermeier, R.A.; Mittermeier, C.G., de Fonseca, G.A.B. \& Kent, J. 2000. Biodiversity and hotspots for conservation priorities. Nature 403: $853-858$.

Naithani, H. B. 1993. Dendrocalamus somdevae: A new species of bamboo from Uttar Pradesh, India. Indian For. 119(6): 504 - 506.

Nautiyal, D.C. \& Gaur, R. D. 1999. A New species of Poa L., family Poaceae from Garhwal Himalaya, India. J. Bombay Nat. Hist. Soc. 96: 285 - 287.

Nayar, M.P. 1996. Hotspots of endemic plants of India, Nepal and Bhutan. Tropical Botanic Garden and Research Institute, Palode, India, pp. 252

Barberá, P.; Quintanar, A.; Peterson, P.M.; Soreng, R.J.; Romaschenko, K. \&Aedo, C. 2019. New combinations, new names, typifications, and a new section, sect. Hispanica, in Koeleria (Poeae, Poaceae). Phytoneuron 46: 1 - 13.

Singh, P.; Karthigeyan, K.; Lakshminarasimhan, P.; \& Dash, S.S. 2015. Endemic Vascular Plants of India. Botanical Survey of India. Calcutta. 
Rekha Yadav et al. 309

Singh, P; Kumari, P. 2018. Endemic bamboos of India- Conservation Status. Botanical Survey of India. Calcutta.

Sekar, K.C. \& Srivastava, S.K. 2005. A New Species of Festuca L. (Poaceae: Pooideae) from India. J. Jap. Bot. 80: 72.

Tiwari, A. P.; Gavade, S.; Lekhak, M. \& Vaishya, J. K. 2018. Lectotypification of two names of Indian Sehima (Poaceae: Andropogoneae). Phytotaxa 349(1): 95-98.

Tripathi, S.; Agnihotri, P; Yadav, R.; Prasad, D; Jaiswal, S \& Husain, T. 2019. A conspectus of the tribe Andropogoneae of Poaceae in Western Himalaya. Pleione. 13(1): 90 - 102. 